\title{
Pembrolizumab monotherapy in advanced NSCLC patients with low PD-L1 expression: is there real evidence?
}

\author{
Alfonso Fiorelli ${ }^{1}$, Fabiana Vitiello ${ }^{2}$, Floriana Morgillo ${ }^{3}$, Mario Santagata ${ }^{4}$, Chiara Spuntarelli ${ }^{4}$, \\ Marina Di Domenico ${ }^{5}$, Mario Santini ${ }^{1}$, Andrea Bianco ${ }^{6}$ \\ ${ }^{1}$ Department of Translational Medical Sciences, Thoracic Surgery Unity, University of Campania "Luigi Vanvitelli", Naples, Italy; ${ }^{2}$ Department of \\ Pneumology and Oncology; Hospital Vincenzo Monaldi, Naples, Italy; ${ }^{3}$ Oncology Unit, ${ }^{4}$ Maxillo and Oro-facial Unit, ${ }^{5}$ General Pathology Unit, \\ ${ }^{6}$ Department of Translational Medical Sciences, Pneumology Unity, University of Campania "Luigi Vanvitelli”, Naples, Italy \\ Correspondence to: Alfonso Fiorelli MD, PhD. Thoracic Surgery Unit, UniversitàdegliStudidella Campania "Luigi Vanvitelli”, Piazza Miraglia, 2, \\ I-80138 Naples, Italy. Email: alfonso.fiorelli@unicampania.it. \\ Comment on: Mok TSK, Wu YL, Kudaba I, et al. Pembrolizumab versus chemotherapy for previously untreated, PD-L1-expressing, locally advanced \\ or metastatic non-small-cell lung cancer (KEYNOTE-042): a randomised, open-label, controlled, phase 3 trial. Lancet 2019;393:1819-30.
}

Submitted May 31, 2019. Accepted for publication Jun 14, 2019.

doi: $10.21037 /$ tcr.2019.06.28

View this article at: http://dx.doi.org/10.21037/tcr.2019.06.28

In the immunotherapy possibilities, pembrolizumab appeared as a leader, along with nivolumab and atezolizumab, in the second-line setting (1). In the last years, the results of phase III KEYNOTE trials (2-6) confirmed the Pembrolizumab as ahead of other checkpoint inhibitors in the first-line setting. Firstly, KEYNOTE-10 (7) showed that the PD-1 inhibitor represented a standard second-line treatment option in NSCLC as it significantly improved OS in comparison with docetaxel following prior platinumbased chemotherapy. Secondly, KEYNOTE-024 (8), which only included patients with PD-L1 Tumor Proportion Score (TPS) $\geq 50 \%$, found that pembrolizumab monotherapy presented a better OS and progression-free survival (PFS) over standard chemotherapy with platinum. Thus, Food and Drug Administration (FDA) and European Medicine Agency (EMA) approved pembrolizumab as first line therapy for advanced NSCLC with TPS $\geq 50 \%$. After the results of KEYNOTE-024 study, the next step was to evaluate whether patients with advanced NSCLC and lower levels of PD-L1 expression (1\% to 49\%) could be treated with first-line pembrolizumab.

Mok et al. (9) planned a randomized, open-label, phase 3 study (KEYNOTE-042) including a cohort of 1.274 patients with previously untreated locally advanced or metastatic NSCLC who had not received prior systemic treatment for metastatic NSCLC and whose tumors did not express EGFR mutation or ALK translocation, but
PD-L1 TPF $\geq 1 \%$. Patients $(n=1,274)$ were randomized in a $1: 1$ to receive pembrolizumab $(n=637)$ or platinumbased chemotherapy $(n=637)$. The first end-point was to evaluate $\mathrm{OS}$ in the subgroup with PD-L1 TPS $\geq 50 \%$, in the subgroup with PD-L1 TPS $\geq 20 \%$, and in the overall population (PD-L1 TPS $\geq 1 \%$ ). Pembrolizumab monotherapy presented better results over chemotherapy in all TPS subgroups, despite greater benefits were associated with higher PD-L1 expression. In the TPS $\geq 1 \%$ population (overall population), pembrolizumab presented a median OS of 16.7 months while chemotherapy a median OS of 12.1 months (HR: 0.81; 95\% CI: 0.71-0.93; $\mathrm{P}=0.0036)$. For the TPS $\geq 20 \%$ subgroup, the median OS was 17.7 months for the pembrolizumab group and 13.0 months for the chemotherapy group (HR:0.77; 95\% CI: $0.64-0.92 ; \mathrm{P}=0.004)$. For the TPS $\geq 50 \%$ subgroup, the estimated median OS was 20 months for patients receiving pembrolizumab and 12.2 months for those receiving chemotherapy (HR: 0.69; 95\% CI: 0.56-0.85; P=0.0006). The comparison of PFS (secondary end-point) or overall response rate between arms in any population showed no significant difference. Median PFS duration among the 3 TPS populations were 7.1 vs. 6.4 months, 6.2 vs. 6.6 months, and 5.4 vs. 6.5 months (significance not formally tested in $\geq 20 \%$ or $\geq 1 \%$ population, since the superiority boundary was not met in $\geq 50 \%$ population). At least 1 subsequent anticancer therapy was received by $38 \%$ of the 
pembrolizumab group and $44 \%$ of the chemotherapy group, including immunotherapy in $3 \%$ and $20 \%$, respectively. Pembrolizumab group compared to chemotherapy group presented lower rate of treatment-related adverse events of any grade $(63 \%$ vs. $90 \%)$ and of grade 3 or worse $(18 \%$ vs. $41 \%$ ), while death considered related to treatment was similar ( $2 \%$ vs. $2 \%)$. Based on the results of KEYNOTE 042, the FDA (10) approved pembrolizumab for first-line treatment for stage III NSCLC patients without EGFR or ALK genomic aberrations and not suitable for surgery and for definitive chemoradiation, and of stage IV NSCLC.

However, as highlighted by other authors $(11,12)$, the KEYNOTE-042 presents several limitations that should be strictly analyzed before drawing definitive conclusions on the real efficacy of pembrolizumab monotherapy in advanced NSCLC patients with low PDL TPS. In the KEYNOTE 042 study population, 599 patients (47\%) had a TPS of $\geq 50 \%$, and 818 (64\%) had a TPS of $\geq 20 \%$, with $35 \%$ vs. $36 \%$ having a TPS of $1 \%$ to $19 \%, 18 \%$ vs. $16 \%$ having a TPS of $20 \%$ to $49 \%$, and $47 \%$ vs. $47 \%$ having a TPS $\geq 50 \%$. Thus, the number of patients with a PD-L1 TPS of $50 \%$ or greater was close to $50 \%$, which is more than $30 \%$ higher than usually seen in the general population. Since the main benefit of treatment was associated with increasing PD-L1 TPS, in theory the results obtained in all three TPS subgroups of the KEYNOTE-042 study population were driven by high PD-L1 expression. An exploratory analysis of the results of KEYNOTE-042 revealed that little survival advantage emerged with pembrolizumab versus chemotherapy in patients with a TPS of $1 \%$ to $49 \%$ (median OS: 13.4 vs. 12.1 months; HR 0.92, 95\% CI: 0.77-1.11). The OS benefit associated with pembrolizumab in KEYNOTE-042 is driven by the high PD-L1 subgroup (TPS $\geq 50 \%$ ) while the benefits are not as clear-cut for those with PD-L1 TPS of $1 \%$ to $49 \%$. Therefore, patients with low PD-L1 expression may benefit from an association between pembrolizumab with standard chemotherapy. This seems to be confirmed by results of the KEYNOTE-189 (13) and KEYNOTE-407 (14) trials showing that pembrolizumab combined with standard first-line platinum chemotherapy presented better OS and PFS than chemotherapy alone independent of PDL1 tumor expression. Thus, patients with PD-L1 TPS $\geq 50 \%$ and without driver mutations should receive first-line pembrolizumab monotherapy, while in patients with lower PD-L1 TPS a pembrolizumab-chemotherapy combination may be likely indicated. The lack of prospective direct comparison does not allow to draw definitive conclusions, thus, the gold-standard treatment should be choice based on patient's clinical condition that may support the decision to add or eliminate chemotherapy.

\section{Acknowledgments}

Funding: None.

\section{Footnote}

Provenance and Peer Review: This article is commissioned and reviewed by the Section Editor Wei Xu (Division of Respiratory Disease, Department of Geriatrics, the First Affiliated Hospital of Nanjing Medical University, Nanjing, China).

Conflicts of Interest: Both authors have completed the ICMJE uniform disclosure form (available at http://dx.doi. org/10.21037/tcr.2019.06.28). The authors have no conflicts of interest to declare.

Ethical Statement: The authors are accountable for all aspects of the work in ensuring that questions related to the accuracy or integrity of any part of the work are appropriately investigated and resolved.

Open Access Statement: This is an Open Access article distributed in accordance with the Creative Commons Attribution-NonCommercial-NoDerivs 4.0 International License (CC BY-NC-ND 4.0), which permits the noncommercial replication and distribution of the article with the strict proviso that no changes or edits are made and the original work is properly cited (including links to both the formal publication through the relevant DOI and the license). See: https://creativecommons.org/licenses/by-nc-nd/4.0/.

\section{References}

1. Fiorelli A, Sagan D, Mackiewicz L, et al. Incidence, Risk Factors, and Analysis of Survival of Unexpected N2 Disease in Stage I Non-Small Cell Lung Cancer. Thorac Cardiovasc Surg 2015;63:558-67.

2. Fiorelli A, Santini M. In lung cancer patients were a malignant pleural effusion is found at operation could resection ever still be justified? Interact Cardiovasc Thorac Surg 2013;17:407-12.

3. Fiorelli, A, Perrotta F, Mollica M, et al. Endoscopic central airway recanalization to enable first line pembrolizumab 
treatment in a PD-L1 strongly positive non-small cell lung cancer: a case report. J Cardiothorac Surg 2019;14:50.

4. Bianco A, Malapelle U, Rocco D, et al. Targeting immune checkpoints in non small cell lung cancer. Curr Opin Pharmacol 2018;40:46-50.

5. Bianco A, Campbell SFM. Atezolizumab plus platinumbased regimen and bevacizumab: is it time to consider immunotherapy in a concurrent approach for lung cancer? Transl Cancer Res 2019;8:S103-5.

6. Perrotta F, Rocco D, Vitiello F, et al. Immune Checkpoint Blockade for Advanced NSCLC: A New Landscape for Elderly Patients. Int J Mol Sci 2019;20. doi: 10.3390/ ijms20092258.

7. Herbst RS, Baas P, Kim DW, et al. Pembrolizumab versus docetaxel for previously treated, PD-L1-positive, advanced non-small-cell lung cancer (KEYNOTE-010): a randomised controlled trial. Lancet 2016;387:1540-50.

8. Reck M, Rodríguez-Abreu D, Robinson AG, et al; KEYNOTE-024 Investigators. Pembrolizumab versus Chemotherapy for PD-L1-Positive Non-Small-Cell Lung Cancer. N Engl J Med 2016;375:1823-33.

Cite this article as: Fiorelli A, Vitiello F, Morgillo F, Santagata M, Spuntarelli C, Di Domenico M, Santini M, Bianco A. Pembrolizumab monotherapy in advanced NSCLC patients with low PD-L1 expression: is there real evidence? Transl Cancer Res 2019;8(Suppl 6):S618-S620. doi: 10.21037/ tcr.2019.06.28
9. Mok TSK, Wu YL, Kudaba I, et al; KEYNOTE-042 Investigators. Pembrolizumab versus chemotherapy for previously untreated, PD-L1-expressing, locally advanced or metastatic non-small-cell lung cancer (KEYNOTE-042): a randomised, open-label, controlled, phase 3 trial. Lancet 2019;393:1819-30.

10. U.S. Food and Drug. Available online: https://www.fda.gov/

11. Smit EF, de Langen AJ. Pembrolizumab for all PD-L1positive NSCLC. Lancet 2019;393:1776-8.

12. Nyberg K. KEYNOTE-042: No Hard and Fast Rules for First-Line Pembrolizumab Regarding PD-L1-Positive Disease. Available online: http://www.lungcancernews.org

13. Gandhi L, Rodríguez-Abreu D, Gadgeel S, et al; KEYNOTE-189 Investigators. Pembrolizumab plus Chemotherapy in Metastatic Non-Small-Cell Lung Cancer. N Engl J Med 2018;378:2078-92.

14. Paz-Ares L, Luft A, Tafreshi A, et al. KEYNOTE-407: phase 3 study of carboplatinpaclitaxel/nab-paclitaxel with or without pembrolizumab for metastatic squamous NSCLC. Paper presented at: American Society of Clinical Oncology Annual Meeting; June 1-5, 2018; Chicago, IL. 